\title{
Hepatoma-Derived Growth Factor: Its Possible Involvement in the Progression of Hepatocellular Carcinoma
}

\author{
Hirayuki Enomoto ${ }^{1, *}$, Hideji Nakamura ${ }^{2}$, Weidong Liu ${ }^{3}$ and Shuhei Nishiguchi ${ }^{1}$ \\ 1 Division of Hepatobiliary and Pancreatic Disease, Department of Internal Medicine, \\ Hyogo College of Medicine, Mukogawa-cho 1-1, Nishinomiya, Hyogo 663-8501, Japan; \\ E-Mail: nishiguc@hyo-med.ac.jp \\ 2 Department of Gastroenterology and Hepatology, Nissay Hospital, Itachibori 6-3-8, Nishi-ku, \\ Osaka 550-0012, Japan; E-Mail: nakamura.hideji@nissay-hp.or.jp \\ 3 Department of Hepatology and Infectious Diseases, the Second Affiliated Hospital, \\ Shantou University Medical College, No. 69, Dongxiabei, Jinping, Shantou 515041, China; \\ E-Mail: weidongliu206@hotmail.com \\ * Author to whom correspondence should be addressed; E-Mail: enomoto@hyo-med.ac.jp; \\ Tel.: +81-798-45-6472.
}

Academic Editor: Tatsuo Kanda

Received: 25 May 2015 / Accepted: 17 June 2015 / Published: 19 June 2015

\begin{abstract}
The development of hepatocellular carcinoma (HCC) is an important complication of viral infection induced by hepatitis virus $C$, and our major research theme is to identify a new growth factor related to the progression of HCC. HDGF (hepatoma-derived growth factor) is a novel growth factor that belongs to a new gene family. HDGF was initially purified from the conditioned medium of a hepatoma cell line. HDGF promotes cellular proliferation as a DNA binding nuclear factor and a secreted protein acting via a receptor-mediated pathway. HDGF is a unique multi-functional protein that can function as a growth factor, angiogenic factor and anti-apoptotic factor and it participates in the development and progression of various malignant diseases. The expression level of HDGF may be an independent prognostic factor for predicting the disease-free and overall survival in patients with various malignancies, including HCC. Furthermore, the overexpression of HDGF promotes the proliferation of HCC cells, while a reduction in the HDGF expression inhibits the proliferation of HCC cells. This article provides an overview of the characteristics of HDGF and describes the potential role of HDGF as a growth-promoting factor for HCC.
\end{abstract}


Keywords: hepatoma-derived growth factor; hepatocellular carcinoma; angiogenesis; apoptosis

\section{Introduction}

The development of hepatocellular carcinoma (HCC) is a major complication of viral infection induced by hepatitis virus $\mathrm{C}[1,2]$. HCC is a common malignant disease, and, despite recent progress in anticancer therapy, patients with advanced HCC continue to show a poor outcome [3,4]. Although several molecules have been determined to be potential targets of anticancer therapy [5], sorafenib, which was designed to inhibit vascular endothelial growth factor (VEGF) signaling, is the only available agent with a clinically demonstrated antitumor effect on HCC [6,7]. Therefore, identifying a new target molecule for HCC treatment is important.

We aimed to discover a new growth factor that may be involved in the progression of HCC. Since the hepatoma-derived cell line Huh-7 autonomously proliferates under serum-free conditions in vitro, we hypothesized the presence of an unknown growth factor in the conditioned medium and succeeded in purifying a new molecule, "hepatoma-derived growth factor (HDGF)" [8,9]. HDGF stimulates the proliferation of hepatoma cells in vitro [10,11], and the HDGF expression is significantly higher in human HCC tissues than in adjacent non-cancerous liver tissues [12]. A high HDGF expression is related to several unfavorable cancer characteristics, including rapid growth, significant invasiveness and metastasis, and it is also associated with poor prognoses of various malignant diseases [13-19].

We initially identified HDGF as a growth-stimulating factor; however, HDGF has also been reported to be an angiogenic factor and probable anti-apoptotic factor. Therefore, this novel molecule may participate in the development and progression of many types of cancer through multiple mechanisms. This article reviews the characteristics of HDGF and describes the potential role of HDGF as a unique growth-promoting factor for HCC.

\section{HDGF as a Novel Unique Growth Factor}

HDGF is a $26-\mathrm{kDa}$ heparin-binding acidic glycoprotein consisting of 240 amino acids that was originally reported as a secreted protein purified from the conditioned medium of Huh-7 hepatoma cells $[8,9]$. Several novel proteins, the N-terminal regions of which are highly homologous to that of HDGF, were subsequently identified, named HDGF-related proteins (HRPs) [20-22]. HDGF and HRPs are considered to form a new gene family, and the highly homologous $\mathrm{N}$-terminal region containing approximately 100 amino acids is represented as the HATH (homologous to the amino terminus of HDGF) domain. Additionally, a survival factor for the lens epithelium, LEDGF (lens epithelium-derived growth factor) [23], is also suggested to be a member of the HDGF family based on the presence of a HATH region in its N-terminus.

Although HDGF proteins are detected in the conditioned media of various types of cells, the sequence of the HDGF protein does not include the N-terminal hydrophobic sequence characteristic of signal peptides. Therefore, HDGF is assumed to be secreted via a pathway that differs from the classical Golgi 
secretion system $[9,24]$. A recent study showed that the 10 amino acids at the N-terminus of HDGF are essential for its secretion. It has also been reported that the phosphorylation of serine 165 in the C-terminal region of HDGF is important for its secretion [25].

Irrespective of the unclarified system(s) of secretion, the exogenous administration of HDGF stimulates the proliferation of various cell types, including benign and malignant cells [26-31]. In addition, the exogenous administration of HDGF enhances the phosphorylation of mitogen-activated protein kinase (MAPK) in gastric epithelial cells and endothelial cells $[32,33]$. Furthermore, exogenously supplied HDGF proteins activate phosphatidylinositol-3 kinase (PI3K)/AKT signaling in NIH3T3 cells [34]. These findings strongly suggest the presence of receptor-mediated signal transduction pathway(s) for HDGF. Recently, part of the HATH region (amino acids 81-100) has been reported to be a possible receptor-binding site [35]. Therefore, the growth-promoting effects of HDGF should at least partially depend on the receptor-mediated signal transduction pathways, such as the intracellular activation of MAPK and/or PI3K/AKT.

In addition to the role of HDGF in receptor-mediated signal transduction, HDGF has two putative nuclear localization signals (NLSs) and it can be transported into the nucleus, suggesting that it may function as a nuclear factor $[10,26]$. The first NLS is located in the HATH region, while the second NLS resides in a gene-specific region (Figure 1). We previously found that nuclear translocation is important for the mitogenic activity of HDGF-overexpressing cells and that the second NLS plays a pivotal role in the growth-stimulating effects of HDGF [10].

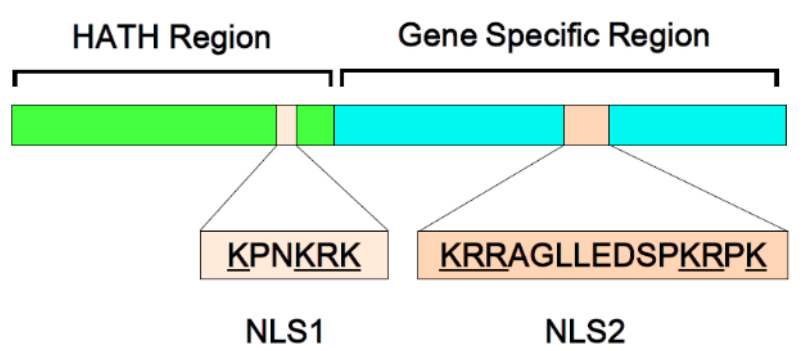

Figure 1. Structure of hepatoma-derived growth factor (HDGF). HDGF protein contains 240 amino acids. The N-terminal region of HDGF protein is highly homologous to that of HDGF-related proteins, and the well-conserved N-terminal amino acid sequence (approximately 100 amino acids) is represented as the HATH (homologous to the amino terminus of HDGF) domain. HDGF has two putative nuclear localization signals (NLSs). The first NLS is a basic amino acid-rich region $\left({ }^{75} \underline{\mathrm{KPNKRK}}{ }^{80}\right.$; basic residues underlined) in the HATH domain (NLS1), and HDGF protein also contains a basic motif $\left({ }^{155} \underline{\mathrm{KRRAGLLEDSPKRPK}}{ }^{170}\right.$; basic residues underlined) in the gene-specific region (NLS2).

Although it is unclear how HDGF stimulates cellular growth after nuclear translocation, previous studies have suggested major roles for the HATH region. The HATH regions of the HDGF family members contain a PWWP motif [36,37] that was initially reported in a candidate gene for Wolf-Hirschhorn syndrome, WHSC1. HDGF binds to a conserved DNA sequence in the promoter region of its target genes and subsequently inhibits their transcription, and the presumed DNA binding site is suggested to be present in the PWWP domain [38]. Therefore, the PWWP motif in the HATH region of HDGF has the potential to function as a DNA binding domain. 
Taken together, HDGF is a unique growth factor with dual mechanisms for promoting cellular proliferation: a receptor-mediated pathway and a direct action mediated by DNA binding following nuclear translocation (Figure 2).

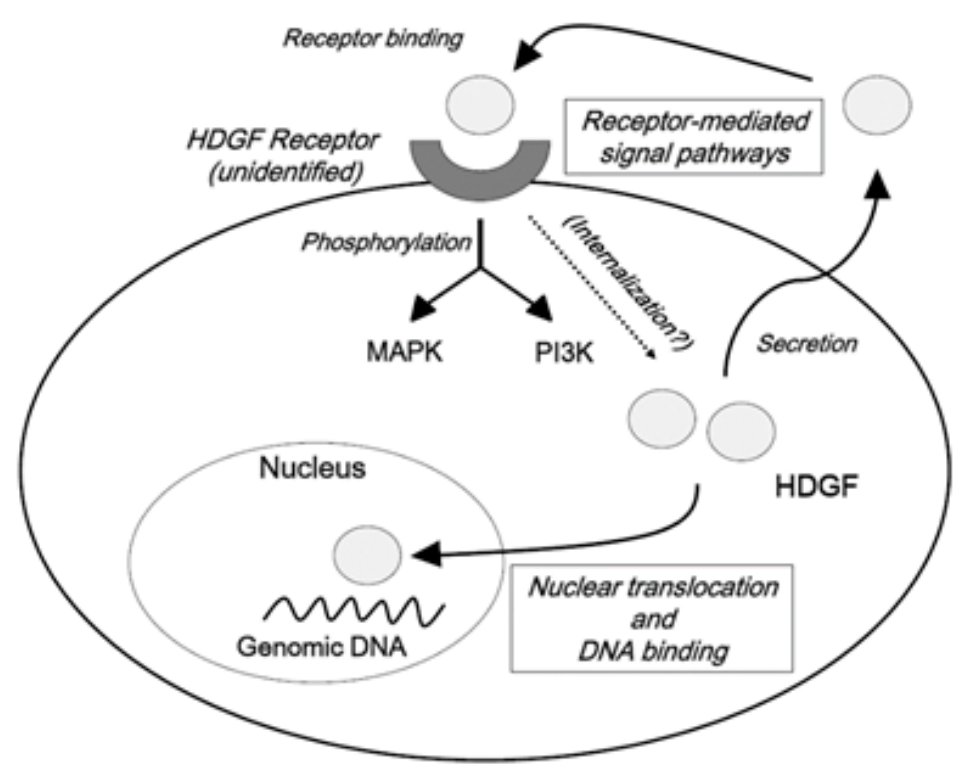

Figure 2. Possible signal pathways of hepatoma-derived growth factor (HDGF). HDGF is a unique growth factor with dual mechanisms for promoting cellular proliferation: a receptor-mediated pathway and a direct action mediated by DNA binding following nuclear translocation. HDGF is considered to be secreted via a pathway that differs from the classical Golgi secretion system. Extracellular HDGF binds to an unidentified receptor and activates MAPK and/or PI3K. HDGF protein also acts as a nuclear protein that regulates the expression of target genes through DNA binding.

\section{HDGF as an Angiogenic Factor}

Although HDGF was isolated from a hepatoma cell line, previous studies have shown that it plays important roles in the development and tissue repair of numerous normal organs, including the liver, kidneys, lungs and gut [27-31]. In addition, Everett et al. [39] reported the functional involvement of HDGF in the development of and tissue repair in the cardiovascular system. We have also shown that HDGF stimulates the proliferation of endothelial cells and that the administration of recombinant HDGF significantly increases tubular formation in experimental vascular formation systems in vitro $[33,40]$. Interestingly, we further demonstrated that the introduction of the HDGF gene in NIH3T3 fibroblasts induces the expression of the most important angiogenic factor VEGF and that the transplantation of HDGF-overexpressing NIH3T3 cells in nude mice results in the development of large and highly vascularized tumors [40]. VEGF is highly expressed in the tumors developing from these HDGF-overexpressing NIH3T3 cells, and the growth of HDGF-overexpressing tumors is partially inhibited by an anti-VEGF neutralizing antibody [40]. In agreement with the findings of our study, HDGF has also been reported to induce VEGF in a gastric cancer cell line [41]. These findings suggest that HDGF may function as an angiogenic factor through two different mechanisms, including its direct effect on endothelial cell proliferation and the induction of VEGF expression. 


\section{HDGF as a Possible Anti-Apoptotic Factor}

The ability to escape apoptotic signals is one of the fundamental mechanisms underlying the survival and uncontrolled growth of malignant cells, and several growth factors are suggested to participate in tumor progression via their anti-apoptotic effects [42]. However, the role of HDGF in the apoptotic pathway remains controversial.

HDGF expression is considered to be related to the increased sensitivity of esophageal cancer cells to radiation therapy [43]. Additionally, the dephosphorylation of HDGF may be involved in caspase-dependent apoptosis under the TNF/cycloheximide-induced apoptosis of endothelial cells [44]. The reduction of HDGF inhibits TNF/cycloheximide-induced apoptosis in HeLa cells [45]. In contrast, several studies have shown an association between HDGF and apoptotic resistance, rather than the induction of apoptosis [46-51]. For instance, HDGF has been reported to be a survival factor for neurons of the central nervous system, motor neurons and olfactory epithelium [46,47]. The reduction of HDGF induces the expression and dephosphorylation of the pro-apoptotic protein Bad and suppresses the ERK-AKT signaling of MAPK, resulting in the activation of the apoptotic pathway [48]. In colorectal cancer cells, the overexpression of HDGF inhibits drug-induced apoptosis, and HDGF knockdown induces apoptosis through the mitochondrial pathway, suggesting that HDGF is involved in the process of cancer cell resistance to chemotherapy [49,50]. Regarding hepatoma cells, blocking HDGF activates both the Fas-mediated extrinsic and Bad-mediated intrinsic apoptotic pathways [48,51], and HDGF is therefore considered to function as a survival factor by exerting multiple anti-apoptotic effects. However, further studies are required before any definitive conclusions can be made regarding the functional role of HDGF in apoptosis, although most recent reports support the anti-apoptotic function of HDGF in various types of cancer cells.

\section{HDGF as a Growth-Promoting Factor for HCC}

HDGF is expressed in several hepatoma cell lines, including Huh-7, HepG2, PLC/PLF/5, SK-Hep1 and Mahlavu [52,53]. The endogenous overexpression of HDGF significantly stimulates the proliferation of hepatoma cells [13]. In a xenograft model using nude mice, HDGF-overexpressing HepG2 hepatoma cells develop larger tumors when compared to the tumors of the control counterparts [52]. In clinical studies, the expression of HDGF in human HCC tissue has been found to be higher than that seen in adjacent non-cancerous tissues [12]. Additionally, HCC patients with a higher HDGF expression have been reported to show earlier recurrence and a poorer overall survival rate compared to patients with lower HDGF expression levels [15,54,55]. A multivariate analysis revealed that high HDGF expression and serum AFP (a well-established tumor marker) levels were independent prognostic factors for the disease-free and overall survival [15]. These findings suggest the significant role of HDGF in the progression of human HCC.

As described above, the HDGF expression has been suggested to contribute to the progression of various malignant diseases. Treatment with HDGF antibodies significantly suppresses the proliferation of lung cancer cells, making HDGF a novel therapeutic target for lung cancer [56]. Meng et al. investigated the effects of HDGF-silencing through shRNA and reported that HDGF may be a therapeutic target for non-small cell lung cancer [57]. Furthermore, several recent experimental studies 
showed HDGF to be associated with the malignant phenotype of cancer cells [58-60]. We previously showed that the reduction of the HDGF expression significantly suppresses the growth of hepatoma cells in vitro $[11,53]$. We recently found that the reduced expression of HDGF inhibits the proliferation of HCC cells in vivo [61]. Since a high HDGF expression is significantly associated with the unfavorable prognosis of various malignant diseases in clinical studies, it is possible that the inhibition of HDGF will provide a new approach for the treatment of malignant diseases, including HCC.

\section{Future Perspectives}

Although HDGF is considered to be a unique growth factor and a potential target molecule for anticancer therapy, the mechanisms by which the HDGF expression is inhibited remain unclear. As a result, more studies must be carried out to obtain new knowledge that is required to develop reliable therapies. Meanwhile, several studies have suggested various mechanisms for regulating the HDGF gene expression. We previously showed that vitamin K2 inhibits the promoter activity of HDGF in hepatoma cells [53]. HDGF may be negatively regulated by the anticancer protein p53 [62]. Interestingly, Shih et al. showed that HDGF is a target gene of microRNA (miR)-214 and that the downregulation of miR-214 contributes to the hypervascularity of HCC via activation of the HDGF paracrine pathway for tumor angiogenesis [63]. In addition, other microRNAs have been reported to affect the HDGF expression. For example, the HDGF expression is upregulated by miR-195 or miR-497 in lung cancer cells $[64,65]$, and miR-141 induces the HDGF expression in gastric cancer cells [66]. Interestingly, using affinity chromatography and proteomic techniques, nucleolin has recently been reported to be a HDGF-interacting membrane protein in hepatoma cells [67].

Nevertheless, the mechanisms underlying the regulation of the HDGF expression and signal transduction must be better understood in order to establish viable anti-HCC strategies.

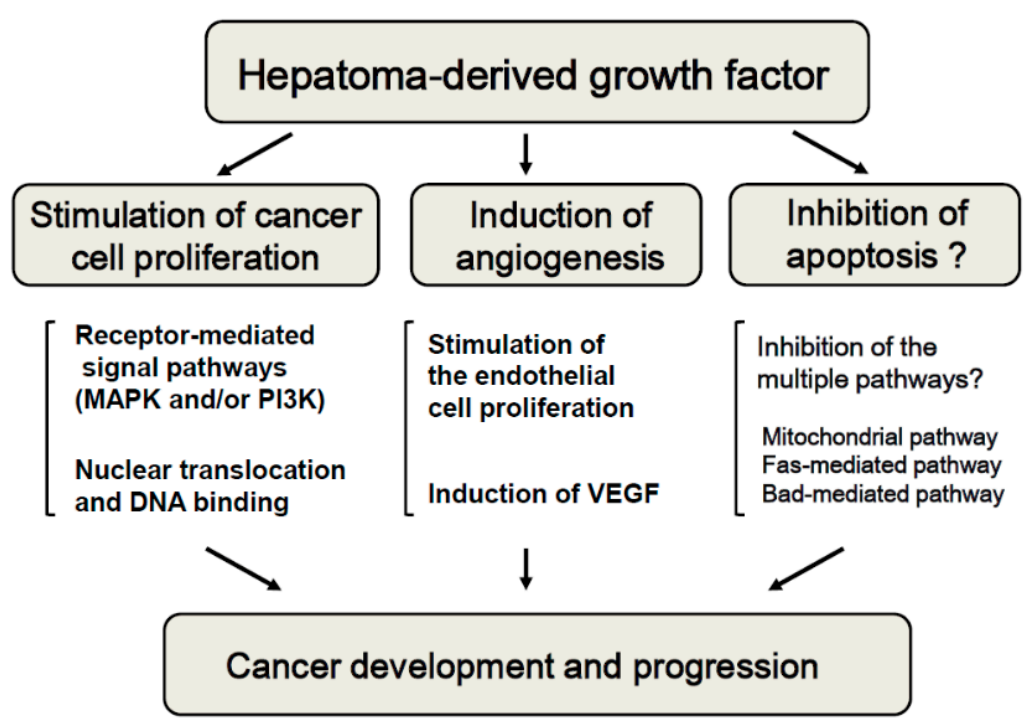

Figure 3. Roles of hepatoma-derived growth factor (HDGF) in the development and progression of malignant diseases. HDGF is a unique multi-functional protein that can function as a growth factor, angiogenic factor and anti-apoptotic factor and it participates in the development and progression of malignant diseases, including HCC. 


\section{Conclusions}

HDGF is a unique molecule with multiple cellular roles. For example, it functions as a growth-stimulating factor, angiogenic factor and possible anti-apoptotic factor (Figure 3). Recent studies have demonstrated the overexpression of HDGF to correlate with poor clinical outcomes in numerous types of malignant diseases. Understanding the regulation of HDGF will thus provide a path for developing novel therapies against HCC.

\section{Acknowledgments}

We thank Yoko Matsushita, Noriko Deguchi, Nozomi Kanazawa and Sayaka Fujii for their technical assistance. This study was supported in part by grants from Grants-in-Aid for Scientific Research (KAKENHI-24590998).

\section{Author Contributions}

All authors participated in the studies. Hirayuki Enomoto and Hideji Nakamura wrote and edited the manuscript. All authors were involved in the manuscript revision and approved the final version of the manuscript.

\section{Conflicts of Interest}

The authors declare no conflict of interest.

\section{Abbreviations}

HCC: hepatocellular carcinoma; VEGF: vascular endothelial growth factor; HDGF: hepatoma-derived growth factor; HRP: HDGF-related protein; HATH: homologous to the amino terminus of HDGF; MAPK: mitogen-activated protein kinase; PI3K: Phosphatidylinositol-3 kinase; NLS: nuclear localization signal; NSCLC: non-small cell lung cancer; miR: microRNA.

\section{References}

1. El-Serag, H.B.; Kanwal, F. Epidemiology of hepatocellular carcinoma in the United States: Where are we? Where do we go? Hepatology 2014, 60, 1767-1775.

2. Ben Ari, Z.; Weitzman, E.; Safran, M. Oncogenic viruses and hepatocellular carcinoma. Clin. Liver Dis. 2015, 19, 341-360.

3. Bruix, J.; Sherman, M. Management of hepatocellular carcinoma: An update. Hepatology 2011, 53, 1020-1022.

4. European Association for the Study of the Liver; European Organisation for Research and Treatment of Cancer. EASL-EORTC clinical practice guidelines: Management of hepatocellular carcinoma. J. Hepatol. 2012, 56, 908-943.

5. Deng, G.L.; Zeng, S.; Shen, H. Chemotherapy and target therapy for hepatocellular carcinoma: New advances and challenges. World J. Hepatol. 2015, 7, 787-798. 
6. Llovet, J.M.; Ricci, S.; Mazzaferro, V.; Hilgard, P.; Gane, E.; Blanc, J.F.; de Oliveira, A.C.; Santoro, A.; Raoul, J.L.; Forner, A.; et al. Sorafenib in advanced hepatocellular carcinoma. N. Engl. J. Med. 2008, 359, 378-90.

7. Cheng, A.L.; Kang, Y.K.; Chen, Z.; Tsao, C.J.; Qin, S.; Kim, J.S.; Luo, R.; Feng, J.; Ye, S.; Yang, T.S.; et al. Efficacy and safety of sorafenib in patients in the Asia-Pacific region with advanced hepatocellular carcinoma: A phase III randomised, double-blind, placebo-controlled trial. Lancet Oncol. 2009, 10, 25-34.

8. Nakamura, H.; Izumoto, Y.; Kambe, H.; Kuroda, T.; Mori, T.; Kawamura, K.; Yamamoto, H.; Kishimoto, T. Molecular cloning of complementary DNA for a novel human hepatoma-derived growth factor. Its homology with high mobility group-1 protein. J. Biol. Chem. 1994, 269, 25143-25149.

9. Nakamura, H.; Enomoto, H. Hepatoma-derived growth factor in carcinogenesis and cancer progression. Curr. Drug Ther. 2011, 6, 278-285.

10. Kishima, Y.; Yamamoto, H.; Izumoto, Y.; Yoshida, K.; Enomoto, H.; Yamamoto, M.; Kuroda, T.; Ito, H.; Yoshizaki, K.; Nakamura, H. Hepatoma-derived growth factor stimulates cell growth after translocation to the nucleus by nuclear localization signals. J. Biol. Chem. 2002, 277, 10315-10322.

11. Kishima, Y.; Yoshida, K.; Enomoto, H.; Yamamoto, M.; Kuroda, T.; Okuda, Y.; Uyama, H.; Nakamura, H. Antisense oligonucleotides of hepatoma-derived growth factor (HDGF) suppress the proliferation of hepatoma cells. Hepatogastroenterology 2002, 49, 1639-1644.

12. Yoshida, K.; Nakamura, H.; Okuda, Y.; Enomoto, H.; Kishima, Y.; Uyama, H.; Ito, H.; Hirasawa, T.; Inagaki, S.; Kawase, I. Expression of hepatoma-derived growth factor in hepatocarcinogenesis. J. Gastroenterol. Hepatol. 2003, 18, 1293-1301.

13. Ren, H.; Tang, X.; Lee, J.J.; Feng, L.; Everett, A.D.; Hong, W.K.; Khuri, F.R.; Mao, L. Expression of hepatoma-derived growth factor is a strong prognostic predictor for patients with early-stage non-small-cell lung cancer. J. Clin. Oncol. 2004, 22, 3230-3237.

14. Guo, S.; Liu, H.D.; Liu, Y.F.; Liu, L.; Sun, Q.; Cui, X.J. Hepatoma-derived growth factor: A novel prognostic biomarker in intrahepatic cholangiocarcinoma. Tumour Biol. 2015, 36, 353-364.

15. Yoshida, K.; Tomita, Y.; Okuda, Y.; Yamamoto, S.; Enomoto, H.; Uyama, H.; Ito, H.; Hoshida, Y.; Aozasa, K.; Nagano, H.; et al. Hepatoma-derived growth factor is a novel prognostic factor for hepatocellular carcinoma. Ann. Surg. Oncol. 2006, 13, 159-167.

16. Bao, C.; Wang, J.; Ma, W.; Wang, X.; Cheng, Y. HDGF: A novel jack-of-all-trades in cancer. Future Oncol. 2014, 10, 2675-2685.

17. Bao, C.H.; Liu, K.; Wang, X.T.; Ma, W.; Wang, J.B.; Wang, C.; Jia, Y.B.; Wang, N.N.; Tan, B.X.; Song, Q.X.; et al. Prognostic role of hepatoma-derived growth factor in solid tumors of Eastern Asia: A systematic review and meta-analysis. Asian Pac. J. Cancer Prev. 2015, 16, 1803-1811.

18. Tsai, C.C.; Huang, S.C.; Tai, M.H.; Chien, C.C.; Huang, C.C.; Hsu, Y.C. Hepatoma-derived growth factor upregulation is correlated with prognostic factors of early-stage cervical adenocarcinoma. Int. J. Mol. Sci. 2014, 15, 21492-21504.

19. Tao, F.; Ye, M.F.; Sun, A.J.; Lv, J.Q.; Xu, G.G.; Jing, Y.M.; Wang, W. Prognostic significance of nuclear hepatoma-derived growth factor expression in gallbladder cancer. World J. Gastroenterol. 2014, 20, 9564-9569. 
20. Izumoto, Y.; Kuroda, T.; Harada, H.; Kishimoto, T.; Nakamura, H. Hepatoma-derived growth factor belongs to a gene family in mice showing significant homology in the amino terminus. Biochem. Biophys. Res. Commun. 1997, 238, 26-32.

21. Ikegame, K.; Yamamoto, M.; Kishima, Y.; Enomoto, H.; Yoshida, K.; Suemura, M.; Kishimoto, T.; Nakamura, H. A new member of a hepatoma-derived growth factor gene family can translocate to the nucleus. Biochem. Biophys. Res. Commun. 1999, 266, 81-87.

22. Dietz, F.; Franken, S.; Yoshida, K.; Nakamura, H.; Kappler, J.; Gieselmann, V. The family of hepatoma-derived growth factor proteins: Characterization of a new member HRP-4 and classification of its subfamilies. Biochem. J. 2002, 366, 491-500.

23. Ge, H.; Si, Y.; Roeder, R.G. Isolation of cDNAs encoding novel transcription coactivators p52 and p75 reveals an alternate regulatory mechanism of transcriptional activation. EMBO J. 1998, 17, 6723-6729.

24. Enomoto, H.; Nakamura, H.; Nishiguchi, S. The Role of Hepatoma-derived Growth Factor (HDGF) in cancer development and progression. Curr. Res. Cancer 2011, 5, 11-25.

25. Thakar, K.; Kröcher, T.; Savant, S.; Gollnast, D.; Kelm, S.; Dietz, F. Secretion of hepatoma-derived growth factor is regulated by N-terminal processing. Biol. Chem. 2010, 391, 1401-1410.

26. Jhaveri, N.; Chen, T.C.; Hofman, F.M. Tumor vasculature and glioma stem cells: Contributions to glioma progression. Cancer Lett. 2014, doi:10.1016/j.canlet.2014.12.028.

27. Kao, Y.H.; Chen, C.L.; Jawan, B.; Chung, Y.H.; Sun, C.K.; Kuo, S.M.; Hu, T.H.; Lin, Y.C.; Chan, H.H.; Cheng, K.H.; et al. Upregulation of hepatoma-derived growth factor is involved in murine hepatic fibrogenesis. J. Hepatol. 2010, 52, 96-105.

28. Gómez, E.; Correia-Álvarez, E.; Caamaño, J.N.; Díez, C.; Carrocera, S.; Peynot, N.; Martín, D.; Giraud-Delville, C.; Duranthon, V.; Sandra, O.; et al. Hepatoma-derived growth factor: From the bovine uterus to the in vitro embryo culture. Reproduction 2014, 148, 353-365.

29. Lepourcelet, M.; Tou, L.; Cai, L.; Sawada, J.; Lazar, A.J.; Glickman, J.N.; Williamson, J.A.; Everett, A.D.; Redston, M.; Fox, E.A.; et al. Insights into developmental mechanisms and cancers in the mammalian intestine derived from serial analysis of gene expression and study of the hepatoma-derived growth factor (HDGF). Development 2005, 132, 415-427.

30. Everett, A.D.; Yang, J.; Rahman, M.; Dulloor, P.; Brautigan, D.L. Mitotic phosphorylation activates hepatoma-derived growth factor as a mitogen. BMC Cell Biol. 2011, 12, doi:10.1186/ 1471-2121-12-15.

31. Mori, M.; Morishita, H.; Nakamura, H.; Matsuoka, H.; Yoshida, K.; Kishima, Y.; Zhou, Z.; Kida, H.; Funakoshi, T.; Goya, S.; et al. Hepatoma-derived growth factor is involved in lung remodeling by stimulating epithelial growth. Am. J. Respir. Cell Mol. Biol. 2004, 30, 459-469.

32. Mao, J.; Xu, Z.; Fang, Y.; Wang, H.; Xu, J.; Ye, J.; Zheng, S.; Zhu, Y. Hepatoma-derived growth factor involved in the carcinogenesis of gastric epithelial cells through promotion of cell proliferation by Erk1/2 activation. Cancer Sci. 2008, 99, 2120-2127.

33. Everett, A.D.; Narron, J.V.; Stoops, T.; Nakamura, H.; Tucker, A. Hepatoma-derived growth factor is a pulmonary endothelial cell-expressed angiogenic factor. Am. J. Respir. Cell Mol. Biol. 2004, 286, L1194-L1201. 
34. Kung, M.L.; Tsai, H.E.; Hu, T.H; Kuo, H.M.; Liu, L.F.; Chen, S.C.; Lin, P.R.; Ma, Y.L.; Wang, E.M.; Liu, G.S.; et al. Hepatoma-derived growth factor stimulates podosome rosettes formation in NIH/3T3 cells through the activation of phosphatidylinositol 3-kinase/Akt pathway. Biochem. Biophys. Res. Commun. 2012, 425, 169-176.

35. Abouzied, M.M.; El-Tahir, H.M.; Prenner, L.; Häberlein, H.; Gieselmann, V.; Franken, S. Hepatoma-derived growth factor. Significance of amino acid residues 81-100 in cell surface interaction and proliferative activity. J. Biol. Chem. 2005, 280, 10945-10954.

36. Stec, I.; Nagl, S.B.; van Ommen, G.J.B.; den Dunnen, J.T. The PWWP domain: A potential protein-protein interaction domain in nuclear proteins influencing differentiation? FEBS Lett. 2000, 473, 1-5.

37. Qiu, C.; Sawada, K.; Zhang, X.; Cheng, X. The PWWP domain of mammalian DNA methyltransferase Dnmt3b defines a new family of DNA-binding folds. Nat. Struct. Biol. 2002, 9 , 217-224.

38. Yang, J.; Everett, A.D. Hepatoma-derived growth factor binds DNA through the N-terminal PWWP domain. BMC Mol. Biol. 2007, 8, 101.

39. Everett, A.D.; Lobe, D.R.; Matsumura, M.E.; Nakamura, H.; McNamara, C.A. Hepatoma-derived growth factor stimulates smooth muscle cell growth and is expressed in vascular development. J. Clin. Investig. 2000, 105, 567-575.

40. Okuda, Y.; Nakamura, H.; Yoshida, K.; Enomoto, H. Uyama, H.; Hirotani, T.; Funamoto, M.; Ito, H.; Everett, A.D.; Hada, T.; et al. Hepatoma-derived growth factor induces tumorigenesis in vivo through both direct angiogenic activity and induction of vascular endothelial growth factor. Cancer Sci. 2003, 94, 1034-1041.

41. Lee, K.H.; Choi, E.Y.; Kim, M.K.; Lee, S.H.; Jang, B.I.; Kim, T.N.; Kim, S.W.; Kim, S.W.; Song, S.K.; Kim, J.R.; et al. Hepatoma-derived growth factor regulates the bad-mediated apoptotic pathway and induction of vascular endothelial growth factor in stomach cancer cells. Oncol. Res. 2010, 19, 67-76.

42. Mohammad, R.M.; Muqbil, I.; Lowe, L.; Yedjou, C.; Hsu, H.Y.; Lin, L.T.; Siegelin, M.D.; Fimognari, C.; Kumar, N.B.; Dou, Q.P.; et al. Broad targeting of resistance to apoptosis in cancer. Semin. Cancer Biol. 2015, doi:10.1016/j.semcancer.2015.03.001.

43. Bao, C.H.; Wang, X.T.; Ma, W.; Wang, N.N.; Un Nesa, E.; Wang, J.B.; Wang, C.; Jia, Y.B.; Wang, K.; Tian, H.; et al. Irradiated fibroblasts promote epithelial-mesenchymal transition and HDGF expression of esophageal squamous cell carcinoma. Biochem. Biophys. Res. Commun. 2015, $458,441-447$.

44. Clermont, F.; Gonzalez, N.S.; Communi, D.; Franken, S.; Dumont, J.E.; Robaye, B. HDGF is dephosphorylated during the early steps of endothelial cell apoptosis in a caspase-dependent way. J. Cell. Biochem. 2008, 104, 1161-1171.

45. Machuy, N.; Thiede, B.; Rajalingam, K.; Dimmler, C.; Thieck, O.; Meyer, T.F.; Rudel, T. A global approach combining proteome analysis and phenotypic screening with RNA interference yields novel apoptosis regulators. Mol. Cell. Proteomics 2005, 4, 44-55.

46. Zhou, Z.; Yamamoto, Y.; Sugai, F.; Yoshida, K.; Kishima, Y.; Sumi, H.; Nakamura, H.; Sakoda, S. Hepatoma-derived growth factor is a neurotrophic factor harbored in the nucleus. J. Biol. Chem. 2004, 279, 27320-27326. 
47. Borders, A.S.; Hersh, M.A.; Getchell, M.L.; van Rooijen, N.; Cohen, D.A.; Stromberg, A.J.; Getchell, T.V. Macrophage-mediated neuroprotection and neurogenesis in the olfactory epithelium. Physiol. Genomics 2007, 31, 531-543.

48. Tsang, T.Y.; Tang, W.Y.; Tsang, W.P.; Kong, S.K.; Kwok, T.T. Downregulation of hepatoma-derived growth factor activates the Bad-mediated apoptotic pathway in human cancer cells. Apoptosis 2008, 13, 1135-1147.

49. Liao, F.; Dong, W.; Fan, L. Apoptosis of human colorectal carcinoma cells is induced by blocking hepatoma-derived growth factor. Med. Oncol. 2010, 27, 1219-1226.

50. Liao, F.; Liu, M.; Lv, L.; Dong, W. Hepatoma-derived growth factor promotes the resistance to anti-tumor effects of nordihydroguaiaretic acid in colorectal cancer cells. Eur. J. Pharmacol. 2010, 645, 55-62.

51. Tsang, T.Y.; Tang, W.Y.; Tsang, W.P.; Co, N.N.; Kong, S.K.; Kwok, T.T. Mechanistic study on growth suppression and apoptosis induction by targeting hepatoma-derived growth factor in human hepatocellular carcinoma HepG2 cells. Cell. Physiol. Biochem. 2009, 24, 253-262.

52. Liu, W.; Nakamura, H.; Deng, H.; Enomoto, H.; Yamamoto, T.; Iwata, Y.; Koh, N.; Saito, M.; Imanishi, H.; Shimomura, S.; et al. A higher expression of hepatoma-derived growth factor in hepatocelular carcinoma cells and more tumor growth in vivo. Trends Cancer Res. 2009, 5, 29-36.

53. Yamamoto, T.; Nakamura, H.; Liu, W.; Cao, K.; Yoshikawa, S.; Enomoto, H.; Iwata, Y.; Koh, N.; Saito, M.; Imanishi, H.; et al. Involvement of hepatoma-derived growth factor in the growth inhibition of hepatocellular carcinoma cells by vitamin K2. J. Gastroenterol. 2009, 44, 228-235.

54. Hu, T.H.; Huang, C.C.; Liu, L.F.; Lin, P.R.; Liu, S.Y.; Chang, H.W.; Changchien, C.S.; Lee, C.M.; Chuang, J.H.; Tai, M.H. Expression of hepatoma-derived growth factor in hepatocellular carcinoma. Cancer 2003, 98, 1444-1456.

55. Zhou, Y.; Zhou, N.; Fang, W.; Huo, J. Overexpressed HDGF as an independent prognostic factor is involved in poor prognosis in Chinese patients with liver cancer. Diagn. Pathol. 2010, 5, doi:10.1186/1746-1596-5-58.

56. Ren, H.; Chu, Z.; Mao, L. Antibodies targeting hepatoma-derived growth factor as a novel strategy in treating lung cancer. Mol. Cancer Ther. 2009, 8, 1106-1112.

57. Meng, J.; Xie, W.; Cao, L.; Hu, C.; Zhe, Z. shRNA targeting HDGF suppressed cell growth and invasion of squamous cell lung cancer. Acta Biochim. Biophys. Sin. 2010, 42, 52-57.

58. Zhao, J.; Ma, M.Z.; Ren, H.; Liu, Z.; Edelman, M.J.; Pan, H.; Mao, L. Anti-HDGF targets cancer and cancer stromal stem cells resistant to chemotherapy. Clin. Cancer Res. 2013, 19, 3567-3576.

59. Li, M.; Shen, J.; Wu, X.; Zhang, B.; Zhang, R.; Weng, H.; Ding, Q.; Tan, Z.; Gao, G.; Mu, J.; et al. Downregulated expression of hepatoma-derived growth factor (HDGF) reduces gallbladder cancer cell proliferation and invasion. Med. Oncol. 2013, 30, doi:10.1007/s12032-013-0587-7.

60. Tsai, H.E.; Liu, G.S.; Kung, M.L.; Liu, L.F.; Wu, J.C.; Tang, C.H.; Huang, C.H.; Chen, S.C.; Lam, H.C.; Wu, C.S.; et al. Downregulation of hepatoma-derived growth factor contributes to retarded lung metastasis via inhibition of epithelial-mesenchymal transition by systemic POMC gene delivery in melanoma. Mol. Cancer Ther. 2013, 12, 1016-1025. 
61. Enomoto, H.; Nakamura, H.; Liu, W.; Iwata, Y.; Nishikawa, H.; Takata, R.; Yoh, K.; Hasegawa, K.; Ishii, A.; Takashima, T.; et al. Reduced expression of hepatoma-derived growth factor inhibits the proliferation of a hepatocellular carcinoma cell line, SK-HEP-1, in vitro and in vivo. 2015, In preparation.

62. Sasaki, Y.; Negishi, H.; Idogawa, M.; Yokota, I.; Koyama, R.; Kusano, M.; Suzuki, H.; Fujita, M.; Maruyama, R.; Toyota, M.; et al. p53 negatively regulates the hepatoma growth factor HDGF. Cancer Res. 2011, 71, 7038-7047.

63. Shih, T.C.; Tien, Y.J.; Wen, C.J.; Yeh, T.S.; Yu, M.C.; Huang, C.H.; Lee, Y.S.; Yen, T.C.; Hsieh, S.Y. MicroRNA-214 downregulation contributes to tumor angiogenesis by inducing secretion of the hepatoma-derived growth factor in human hepatoma. J. Hepatol. 2012, 57, 584-591.

64. Guo, H.; Li, W.; Zheng, T.; Liu, Z. miR-195 Targets HDGF to inhibit proliferation and invasion of NSCLC cells. Tumour Biol. 2014, 35, 8861-8866.

65. Zhao, W.Y.; Wang, Y.; An, Z.J.; Shi, C.G.; Zhu, G.A.; Wang, B.; Lu, M.Y.; Pan, C.K.; Chen, P. Downregulation of miR-497 promotes tumor growth and angiogenesis by targeting HDGF in non-small cell lung cancer. Biochem. Biophys. Res. Commun. 2013, 435, 466-471.

66. Chen, B.; Huang, T.; Jiang, J.; Lv, L.; Li, H.; Xia, S. miR-141 suppresses proliferation and motility of gastric cancer cells by targeting HDGF. Mol. Cell. Biochem. 2014, 388, 211-218.

67. Chen, S.C.; Hu, T.H.; Huang, C.C.; Kung, M.L.; Chu, T.H.; Yi, L.N.; Huang, S.T.; Chan, H.H.; Chuang, J.H., Liu, L.F.; et al. Hepatoma-derived growth factor/nucleolin axis as a novel oncogenic pathway in liver carcinogenesis. Oncotarget 2015, in press.

(C) 2015 by the authors; licensee MDPI, Basel, Switzerland. This article is an open access article distributed under the terms and conditions of the Creative Commons Attribution license (http://creativecommons.org/licenses/by/4.0/). 